\title{
Teratoma extragonadal del omento mayor, una localización poco usual: reporte de caso y revisión de la literatura
}

\author{
Extragonadal teratoma of the greater omentum, an unusual location: \\ case report and review of the literature
}

\author{
Juan C. Arenas $\mathbb{D}^{\mathbb{D}}$, Julián A. Romo² $\mathbb{D}$, Laura A. López ${ }^{3} \mathbb{D}$
}

1 Médico, especialista en Cirugía General y Epidemiología, Departamento de Cirugía General, Hospital Universitario Mayor Méderi; profesor asistente, Escuela de Medicina y Ciencias de la Salud, Universidad del Rosario, Bogotá, D.C., Colombia.

2 Médico, especialista en Cirugía General, Departamento de Cirugía General, Hospital Universitario Mayor Méderi, Bogotá, D.C., Colombia.

3 Médica interna, Escuela de Medicina y Ciencias de la Salud, Universidad del Rosario. Hospital Universitario Mayor Méderi, Bogotá, D.C., Colombia.

\section{Resumen}

Introducción. Los teratomas son masas de células totipotenciales, frecuentes en los ovarios o testículos. Su localización extragonadal es rara, siendo el epiplón aún más infrecuente. Hasta la actualidad se conocen aproximadamente 34 casos similares en la literatura.

Presentación del caso. Se presenta una paciente de 6I años, con dolor en hemiabdomen inferior asociado a sensación de masa, con marcadores tumorales negativos e imágenes diagnósticas que muestran una masa encapsulada y calcificación anular interna sugestiva de necrosis grasa antigua versus tumor desmoide. Por la presentación clínica y los hallazgos imagenológicos se decide llevar a laparotomía exploratoria donde se encuentra masa quística dependiente de epiplón, con material sebáceo en su interior entremezclado con pelo y fragmentos óseos, que se reseca en bloque sin complicaciones. El informe del estudio histopatológico finalmente concluyó la presencia de un teratoma quístico maduro con cambios degenerativos. Por evolución satisfactoria se da egreso hospitalario.

Discusión. El teratoma quístico extragonadal del epiplón es exótico en la literatura mundial, con escasos reportes de casos. En este caso, la resección mediante laparotomía resultó exitosa y sin complicaciones. Por su baja incidencia, la laparotomía ha sido una opción válida y segura para el manejo, no obstante conocer el caso permite plantear un abordaje con técnicas mínimamente invasivas.

Palabras clave: teratoma; epiplón; quiste dermoide; neoplasias abdominales.

Fecha de recibido: 16/08/2019 - Fecha de aceptación: 04/12/2019

Correspondencia: Laura A. López, Calle 175 No 6-60, torre 4, apartamento 303, Bogotá, D.C., Colombia. Teléfono: (+57) 3046495051

Correo electrónico: alejandralopez236@gmail.com

Citar como: Arenas JC, Romo JA, López LA. Teratoma extragonadal del omento mayor, una localización poco usual: reporte de caso y revisión de la literatura. Rev Colomb Cir. 2020;35:689-94. https://doi.org/10.30944/20117582.452

Este es un artículo de acceso abierto bajo una Licencia Creative Commons - BY-NC-ND https://creativecommons.org/licenses/by-nc$\mathrm{nd} / 4.0 /$ deed.es 


\begin{abstract}
Introduction. Teratomas are totipotential cell masses, commonly located in the ovaries or testes. Its extragonadal location is rare, in the omentum being even rarer. To date, approximately 34 similar cases are known in the literature.

Case presentation. A 6I-year-old female patient is presented, with pain in the lower abdomen associated with a sensation of mass, with negative tumor markers and diagnostic images that show an encapsulated mass and internal annular calcification suggestive of old fat necrosis versus desmoid tumor. Due to the clinical presentation and the imaging findings, it was decided to carry out exploratory laparotomy where a cystic mass dependent on the omentum was found, with sebaceous material intermixed with hair and bone fragments, which was resected in bloc without complications. The histopathological study report finally concluded the presence of a mature cystic teratoma with degenerative changes. Due to satisfactory evolution, he was discharged from hospital.

Discusion. Extragonadal cystic teratoma of the omentum is exotic in the world literature, with few case reports. In this case, the laparotomy resection was successful and uncomplicated. Due to its low incidence, laparotomy has been a valid and safe option for management, although knowing the case allows us to propose an approach with minimally invasive techniques.
\end{abstract}

Keywords: teratoma; omentum; dermoid cyst; abdominal neoplasms.

\section{Introducción}

Los teratomas son masas que surgen de células totipotenciales, contienen tejidos derivados de al menos dos líneas germinales (ectodermo, endodermo o mesodermo), aparecen a cualquier edad y su ubicación más frecuente son los ovarios y los testículos ${ }^{1,2}$. Su localización extragonadal es rara, siendo el epiplón aún más infrecuente.

El primero en reportar un caso de teratoma del omento mayor fue Lebert en I734, quien encontró un tumor que contenía pelo y dientes en el omento de una niña de 15 años ${ }^{3}$. Hasta la actualidad han sido reportados aproximadamente 34 casos similares, ninguno en Colombia. Su etiología es incierta, sin embargo, existen diferentes hipótesis sobre su origen ${ }^{4,5}$. Generalmente los teratomas extragonadales (TEG) son asintomáticos, pero pueden presentarse con un cuadro clínico insidioso ${ }^{3}$. El manejo es predominantemente quirúrgico y los teratomas malignos pueden requerir manejo adicional ${ }^{6}$. En este reporte se presenta el caso de una paciente mayor con un teratoma quístico maduro del omento mayor sometido a manejo quirúrgico.

\section{Caso clínico}

Se trata de una paciente de 6I años, con antecedentes de accidente cerebrovascular de tipo isquémico con posterior hemiparesia izquierda y miomatosis uterina asociada a hemorragia uterina anormal, quien consulta por cuadro clínico de 5 años de evolución consistente en dolor en hemiabdomen inferior, asociado a presencia de masa abdominal. Había sido estudiada en otra institución por medio de resonancia magnética que mostró lesión nodular de $95 \times 75 \times 73 \mathrm{~mm}$ y contornos lobulados, con anillo hipointenso con tenue captación capsular, de contenido hiperintenso, que genera compresión extrínseca del domo vesical, consistente con posible masa anexial.

Por sospecha de lesión de origen ginecológico es valorada por los servicios de ginecología y gineco-oncología, quienes ampliaron el estudio de la masa con marcadores tumorales (antígeno carcinoembrionario $0,79 \mathrm{ng} / \mathrm{ml}$, alfa fetoproteína I,64 ng/ml y CA I25 I2,3 U/ml) los cuales se encontraron dentro de límites normales. En adición se realizó tomografía computarizada (TC) de abdomen (figura I) que mostró calcificación en 


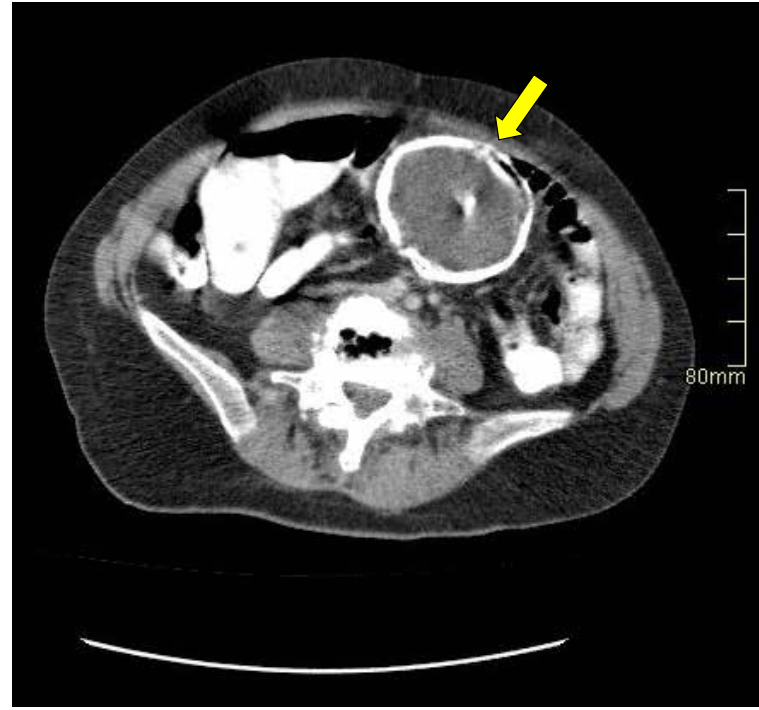

Figura 1. Tomografía computarizada de abdomen que documenta masa intraabdominal (flecha amarilla) en íntimo contacto con tejidos retroperitoneales.

el fondo uterino, probablemente por miomatosis calcificada y una masa de densidad negativa (grasa) encapsulada con calcificación anular alrededor de la misma, localizada en el mesenterio con medidas de $70 \times 66 \times 73 \mathrm{~mm}$, por lo cual se consideró posible la presencia de necrosis grasa encapsulada antigua versus una neoplasia primaria del mesenterio, como tumor desmoide.

Por la persistencia del dolor abdominal asociado a los hallazgos en estudios diagnósticos se interconsulta a cirugía general. Se decidió llevar a cirugía por medio de laparotomía exploratoria en la que se encontró una masa de consistencia pétrea (figura 2), con adherencias firmes y laxas, dependiente de epiplón mayor y cuerno izquierdo de vejiga, sin líquido ascítico ni siembras peritoneales macroscópicas en el resto de la cavidad abdominal. Se resecó en bloque sin ningún inconveniente. El informe histopatológico finalmente concluyó la presencia de un teratoma quístico maduro con cambios degenerativos, con paredes calcificadas y con abundante material sebáceo en su interior, entremezclado con pelo y fragmentos de tejido óseo (figura 3). La paciente presentó evolución postoperatoria lenta hacia la mejoría, con presencia de íleo postoperatorio

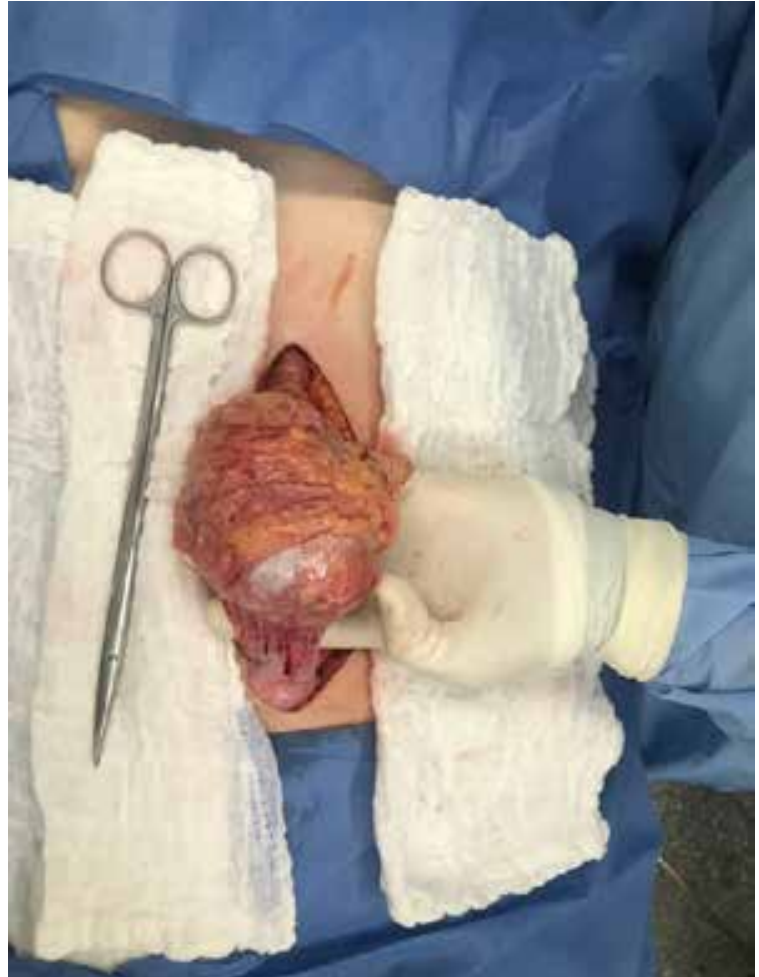

Figura 2. Teratoma dependiente del omento mayor.

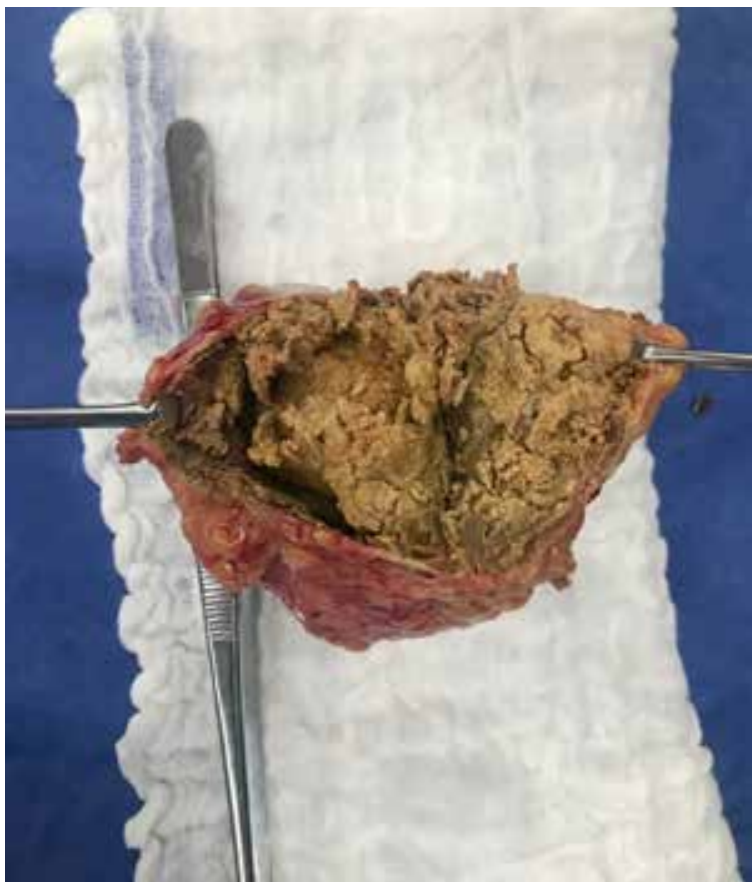

Figura 3. Al interior del teratoma se observa pelo y material sebáceo. 
que se trató con manejo médico, con posterior resolución y egreso, con un tiempo de estancia hospitalaria de 18 días. En el control ambulatorio se verifica evolución satisfactoria.

\section{Discusión}

Los teratomas extragonadales (TEG) son bastante raros, reportándose una incidencia de apenas 0,4 $\%$ entre todos los teratomas ${ }^{6,7}$. Constituyen la segunda neoplasia más común en niños después de los tumores del saco vitelino; en adultos son raros y se presentan en su mayoría en mujeres de mediana edad ${ }^{1,6}$. De manera general, los teratomas pueden tener grados variables de diferenciación, de allí la subclasificación en maduros e inmaduros ${ }^{2}$.

Los teratomas maduros contienen tejidos diferenciados de cualquiera de las tres líneas celulares germinales, formando así teratomas quísticos maduros cuando poseen tejidos de las tres capas, o quistes dermoides cuando se forman a partir de dos capas ${ }^{2,6,8}$. Pueden incluir pelo, dientes y otros derivados ectodérmicos. Se comportan como masas sólidas de bajo grado de malignidad, pero pueden crecer hasta ser irresecables ${ }^{1,6}$. Por otra parte, los teratomas inmaduros están formados por líneas menos diferenciadas; contienen elementos completamente diferenciados con tejidos de apariencia fetal. En adultos, la presencia de elementos inmaduros puede relacionarse con comportamiento maligno de mal pronóstico $^{2,6}$.

Los TEG se originan típicamente en la línea media y varían según la edad, siendo las localizaciones más frecuentes sacrococcígea $(67 \%)$, cabeza y cuello (9\%), retroperitoneo (8\%), mediastino $(7 \%)$, cerebro o medula espinal (6\%), hígado $(\mathrm{I}, 5 \%)$ y pared abdominal $(\mathrm{I}, 5 \%)^{6}$. El mediastino es el sitio más común en adultos ${ }^{9-12}$. La localización exclusiva abdominal o retroperitoneal es rara, comprendiendo menos del $5 \%$ de todos los TEG, y su localización en el epiplón es aún más inusual ${ }^{3,7,13}$.

Los teratomas localizados a nivel del omento tienen un origen incierto. Durante el desarrollo fetal las células migran del saco vitelino hacia el intestino primitivo y hacia las crestas urogenitales, y dichas células totipotenciales pueden dar origen a varios tejidos de las tres capas ${ }^{5,14}$. Existen tres teorías que explican la presencia de TEG en el omento mayor:

I. A partir de células germinales desplazadas que durante la migración se detienen a nivel del mesenterio dorsal, que posteriormente forma el omento mayor ${ }^{4,5}$.

2. A partir de ovarios supernumerarios en el omento, ya sea por atrapamiento de las células germinales en el mesenterio dorsal con posterior diferenciación como estroma ovárico o por desprendimiento y trasplantación en el mesenterio dorsal con posterior desarrollo de un ovario ectópico ${ }^{4,15}, \mathrm{y}$

3. Por auto-amputación de un quiste dermoide del ovario con posterior implantación en el omento mayor, como resultado de torsión subaguda o crónica del pedículo, que produce adhesión a estructuras adyacentes y generación de nueva circulación colateral 4,5,13,16.

Los TEG suelen encontrarse de forma incidental como una masa sin síntomas asociados; en algunos pacientes pueden presentarse con dolor lumbar o abdominal, síntomas genitourinarios o gastrointestinales, edema genital o de miembros inferiores por compresión del drenaje linfático o parestesias en miembros inferiores ${ }^{3,17}$. Pueden ocurrir complicaciones como obstrucción intestinal, hemorragia, infección, transformación maligna o ruptura que produce peritonitis química $^{6,8,17,18}$.

El diagnóstico preoperatorio es complejo. Tanto los teratomas maduros como los inmaduros se asocian a marcadores tumorales normales; a diferencia de otros tumores del saco vitelino, no hay elevación de alfa-fetoproteína ${ }^{\mathrm{I}, \mathrm{I} 9}$. Pueden expresarse otros marcadores relacionados con la regulación del crecimiento celular que podrían asociarse con su comportamiento y respuesta a quimioterapia ${ }^{\mathrm{I}, \mathrm{I}}$. Es factible visualizar calcificaciones en la radiografía de abdomen ${ }^{17}$. La ecografía muestra áreas quísticas con septos y 
áreas sólidas ${ }^{1,19}$. La TC es el método diagnóstico de elección, ya que muestra paredes delgadas con lesiones sólidas, grasa, calcificaciones y zonas atenuadas consistentes con queratina ${ }^{6,14}$. El desplazamiento entre el contenido graso y acuoso es una característica en la resonancia magnética. Una pared engrosada con un margen irregular puede ser un signo de transformación maligna ${ }^{6}$.

El estudio histopatológico es el estándar para el diagnóstico ${ }^{1,6}$. Está descrito el aspirado con aguja fina para la caracterización de TEG en caso de que esté contraindicada la biopsia excisional, no obstante, el tratamiento requiere intervención quirúrgica ${ }^{6,10}$. El abordaje actual abarca diversas técnicas mínimamente invasivas incluyendo laparoscopia de un solo puerto y técnicas robóticas ${ }^{20,21}$. La resección incompleta provoca recurrencia y otras complicaciones ${ }^{18}$.

Los teratomas benignos tienen buen pronóstico sólo con resección, mientras que los teratomas inmaduros deben tratarse como potencialmente malignos ${ }^{4}$. Los teratomas con transformación maligna tienen pobre pronóstico, lo que puede prevenirse removiendo quirúrgicamente el teratoma ${ }^{2}$. Los carcinomas escamosos, tumores carcinoides y adenocarcinomas son las lesiones malignas más frecuentes que provienen de teratomas ${ }^{9,10,22}$. En dichos casos se usa el tratamiento quirúrgico con posterior quimioterapia o radioterapia ${ }^{6}$.

\section{Conclusiones}

Los TEG son tumores infrecuentes y de pronóstico variable según el grado de diferenciación, no obstante, deben tenerse en cuenta a la hora de proponer diagnósticos diferenciales en pacientes con masas intraperitoneales, especialmente en pacientes de mediana edad, como en el caso presentado. Aunque el diagnóstico definitivo se establece con el estudio histopatológico de la masa, es posible pensar antes de la cirugía en un TEG como diagnóstico, teniendo en cuenta que la imagen de elección es la TC y que los marcadores tumorales son negativos en la mayoría de los casos. En el caso presentado, la resección mediante laparotomía resultó exitosa y sin com- plicaciones. Por su baja incidencia, el abordaje abierto es una opción válida y segura para el manejo, aunque se pueden plantear abordajes con técnicas mínimamente invasivas.

\section{Consideraciones éticas}

La aprobación ética del protocolo de investigación fue eximida por el Comité de Ética en Investigación de la Universidad del Rosario (CEI-UR) del I7 de enero de 20I9, acta No 380.

Consentimiento informado: Se obtuvo el consentimiento informado por escrito de la paciente para la publicación de este reporte de caso y sus imágenes adjuntas. El nombre, datos de identificación, historia clínica e imágenes de la paciente serán sometidos a absoluta reserva y permanecerán bajo custodia del Hospital Universitario Mayor Méderi y la Universidad del Rosario.

Conflicto de interés: Los autores no declaran ningún conflicto de interés respecto al presente artículo.

Fuente de financiación: Recursos propios de los autores.

\section{Referencias}

I. Wetherell D. Mature and immature teratoma: A review of pathological characteristics and treatment options. Med Surg Urol. 2014;3:I. https://doi.org/IO.4I72/2168-9857.IO00I24

2. Shafaee MN, Tannir NM, Pagliaro LC. Testicular cancer. En: Kantarjian HM, Wolff RA. The MD Anderson Manual of medical oncology. 3rd ed. New York: McGraw-Hill; 20I8. Fecha de consulta: 2I de febrero de 20I9. Disponible en: http://accessmedicine.mhmedical. com/content.aspx?bookid=I772\&sectionid=I2190I468

3. Mumey N. Dermoid cysts of the great omentum. Am J Surg. I928;5:56-60. Fecha de consulta: 2I de febrero de 20I9. Disponible en: http://linkinghub.elsevier.com/ retrieve/pii/Sooo2961028903190

4. Ushakov F. Parasitic ovarian dermoid tumor of the omentum - A review of the literature and report of two new cases. Eur J Obstet Gynecol Reprod Biol. 1998;8I:7782. https://doi.org/IO.IOI6/sO3OI-2II5(98)ool44-4.

5. Hegde P. Extragonadal omental teratoma: A case report. J Obstet Gynaecol Res. 2014;40:6I8-2I. https://doi.org/IO.IIII/jog.I2I98 
6. O’Donovan EJ, Thway K, Moskovic EC. Extragonadal teratomas of the adult abdomen and pelvis: a pictorial review. Br J Radiol. 20I4;87:20140II6.

https://doi.org/IO.I259/bjr.20I40II6

7. Bernal-Martínez S, Vaca-Carvajal GJ, Arrazola-González JA. Quiste dermoide parasítico en el epiplón. Reporte de un caso. Ginecol Obstet Mex. 20I6;84:53-9. Fecha de consulta: 2I de febrero de 20I9. Disponible en: https:/ginecologiayobstetricia.org.mx/secciones/ casos-clinicos/quiste-dermoide-parasitico-en-el-epiplon-reporte-de-un-caso/

8. Hosokawa T, Sato Y, Seki T, Maebara M, Ito K, Kuribayashi S. Malignant transformation of a mature cystic teratoma of the ovary with rupture. Jpn J Radiol. 2010;28:372-5.

https://doi.org/I0.I007/sir604-0I0-0434-0

9. McKenney JK, Heerema-McKenney A, Rouse RV. Extragonadal germ cell tumors: A review with emphasis on pathologic features, clinical prognostic variables, and differential diagnostic considerations. Adv Anat Pathol. 2007;14:69-92.

https://doi.org/Io.I097/PAP.oboi3e31803240e6.

Io. Gupta R, Mathur SR, Arora VK, Sharma SG. Cytologic features of extragonadal germ cell tumors. Cancer. 2008;II4:504-II. https://doi.org/IO.IOO2/cncr.23983

II. Román-González A, Vélez-Hoyos A, Jaller A, Rúa-Marín C. Teratoma de tiroides, reporte de caso. Rev Colomb Endocrinol Diabetes y Metab. 20I6;3:48-50.

I2. Cortés-Caballero C, Duarte-Vergara LM, Parada-Álvarez MF, Vega-García JM, Vera-Campos JA. Teratoma retroperitoneal en niños: revisión del tema a propósito de un caso. Iatreia. 2014;27:189-97.

13. Kearney MS. Synchronous benign teratomas of the greater omentum and ovary. BJOG An Int J Obstet Gynaecol. 1983;90:676-9.

https://doi.org/IO.IIII/j.I47I-0528.I983.tbo9290.x

I4. Ali AA, Sall I, El Kaoui H, Bouchentouf SM, El Hjjouji A, El Fahssi M, et al. Teratoma of the greater omentum. Can J Surg. 2009;52:E54-5. Fecha de consulta: 2I de febrero de 20I9. Disponible en: http://www.ncbi.nlm.nih. gov/pubmed/19503646
I5. Furuhashi M, Katsumata Y, Oda H, Imai N. Cystic teratoma of the greater omentum: A case report and review of the literature. J Obstet Gynaecol Res. 1997;23:359-63. https://doi.org/IO.IIII/j.I447-0756.I997.tboo858.x

I6. Tokunaga M, Seta M, Yamada M, Nishio M, Yamamoto $\mathrm{K}$, Koyasu Y. Coexistent dermoid cysts of the pouch of the Douglas and ovary resected by laparoscopy. Asian J Endosc Surg. 2012;5:3I-3. https://doi.org/IO.IIII/j.I7585910.2011.00107.X

I7. Wani BN, Rathod V, Banode P, Bhole A. An omental teratoma in a young girl. Clin Pract. 20II;I:eI29. https://doi.org/I0.408I/cp.20II.eI29

18. Shamshirsaz AA, Shamshirsaz AA, Vibhakar JL, Broadwell C, Van Voorhis BJ. Laparoscopic management of chemical peritonitis caused by dermoid cyst spillage. J Soc Laparoendosc Surg. 2011;15:403-5. https://doi.org/IO.4293/I086808IIXI3I25733356990

19. Walsh C, Rushton HG. Diagnosis and management of teratomas and epidermoid cysts. Urol Clin North Am. 2000;27:509-I8. https://doi.org/Io.IOI6/s0094-0I43(05)70098-7

20. Willems E, Martens S, Beelen R. Robotically enhanced mediastinal teratoma resection: a case report and review of the literature. Acta Chir Belg. 2016;II6:309-I2. https://doi.org/Io.IO8o/oool5458.2016.II47264

2I. Takeda A, Imoto S, Mori M, Yamada J, Nakamura H. Early abdominal pregnancy complicated by parasitic dermoid cyst: Diagnosis by diffusion-weighted magnetic resonance imaging and management by laparoendoscopic single-site surgery. J Minim Invasive Gynecol. 2012;19:647-50. https://doi.org/IO.IOI6/j.jmig.20I2.03.024

22. Pacheco JO, Abello V, Omaña OP. Asociación entre tumor de células germinales y malignidades hematológicas. Repert. Med. Cir. 2015;24:69-72. Fecha de consulta: 3 de diciembre de 20I9. Disponible en: https:// revistas.fucsalud.edu.co/index.php/repertorio/article/ view/66o 